\title{
A!
}

This is an electronic reprint of the original article.

This reprint may differ from the original in pagination and typographic detail.

Motlagh, N. H.; Bagaa, M.; Taleb, T.

\section{Energy and Delay Aware Task Assignment Mechanism for UAV-based loT Platform}

Published in:

IEEE Internet of Things Journal

DOI:

10.1109/JIOT.2019.2907873

Published: 01/01/2019

Document Version

Peer reviewed version

Please cite the original version:

Motlagh, N. H., Bagaa, M., \& Taleb, T. (2019). Energy and Delay Aware Task Assignment Mechanism for UAVbased loT Platform. IEEE Internet of Things Journal, 6(4), 6523 - 6536.

https://doi.org/10.1109/JIOT.2019.2907873

This material is protected by copyright and other intellectual property rights, and duplication or sale of all or part of any of the repository collections is not permitted, except that material may be duplicated by you for your research use or educational purposes in electronic or print form. You must obtain permission for any other use. Electronic or print copies may not be offered, whether for sale or otherwise to anyone who is not an authorised user. 
This is the accepted version of the original article published by IEEE.

(C) 2019 IEEE. Personal use of this material is permitted. Permission from IEEE must be obtained for all other uses, in any current or future media, including reprinting/republishing this material for advertising or promotional purposes, creating new collective works, for resale or redistribution to servers or lists, or reuse of any copyrighted component of this work in other works. 


\title{
UAV Selection for a UAV-based Integrative IoT Platform
}

\author{
Naser Hossein Motlagh, Miloud Bagaa and Tarik Taleb \\ Dep. of Communications and Networking, School of Elect Eng, Aalto University, Espoo, Finland. \\ Emails: naser.hossein.motlagh@aalto.fi \\ miloud.bagaa@aalto.fi \\ tarik.taleb@aalto.fi
}

\begin{abstract}
This paper, presents a UAV-based integrative IoT platform that leverages UAVs to deliver different IoT services from height. One of the major tasks of the platform is to select the appropriate UAVs. This selection may be based on different criteria, such as UAVs equipment, energy budget, geographical proximity of the UAV to the area of interest, etc. For the selection mechanism, this paper proposes and formulates two Linear Integer Problem (LIP) optimization solutions by aiming at minimizing the energy consumption and shortening the UAV operation time. For the solutions, Energy Aware Selection of UAVs (EAS) and Delay Aware Selection of UAVs (DAS) are evaluated through simulation. The results show that if the objective is the energy, EAS is more efficient than DAS in case of total energy consumption by the UAVs. Additionally, if the time is the objective, DAS has higher performance than EAS in case of operation time.
\end{abstract}

Index Terms-Unmanned Aerial Vehicle (UAV), UAV/Drone Selection Mechanism, and UAV-based IoT Platform.

\section{INTRODUCTION}

For data communications, Internet of Things (IoT) utilize intelligent interfaces for connecting devices, machines, smart objects, smart environments, services, and persons at anytime, anywhere ideally using any network and any service [1]. These devices not only are installed on the earth, even many of them can be installed as Unmanned Aerial Vehicles (UAVs; alternatively known as drones) payload to provide IoT services from the sky whenever needed. UAVs are in different shapes and sizes, such as fixed wings or multirotors. Depending on the target application, they are broadly categorized in three classes [2]. Micro and Mini, Tactical, and Strategic UAVs. Micro and Mini UAVs are the smallest ones in size and fly at a low altitude, specifically below 300 meters. They are mostly used for civil and commercial applications. The applications of UAVs are divers. For instance, they are applied in agriculture, search and rescue operations, forestry, ocean and lakes, and oil and gas industry. For Using UAVs, they must be equipped with IoT devices such as different sensor types and cameras.

The deployment of UAV applications leads to very large economic benefits for many companies. Amazon and Finnish Post Ltd, DHL Parcel Copter, and German Cargo Drones are good examples to name as they are using the UAVs for parcel delivery [3]. There are many applications of UAVs and yet the market of the use of UAVs is in its own starting phase. A recent study expects global spending on UAVs to nearly double from 5.2 in 2013 to 11.6 billion USD by 2023 [4].
In this paper we propose a UAV selection mechanism for a UAV-based integrative IoT platform. To the best of our knowledge, this problem has not been studied in the literature. For this reason in the next section we intend to describe our proposed UAV communication platform. For the UAV selection mechanism, we propose two Linear Integer Problem to deal the problem of UAV selection. The first optimization aims at minimizing the energy consumption as much as possible in order to handle an event. This solution aims to ensure a fair energy consumption among different UAVs. Meanwhile, the second optimization problem aims at minimizing the time to handle an event i.e. operation time regardless of the energy consumption. The performance of these proposed solutions are validated through simulations.

The rest of the paper is organized as follows. Section II describes our proposed communication network. Section III depicts the UAV selection mechanism and formulation. Section IV provides the optimal solutions for UAV selection and presents the simulation results. Finally, Section V concludes the paper.

\section{UAV COMMUNICATION PLATFORM}

The basic task of a UAV is collecting data from the remote locations. This data collection system requires selecting suitable equipment such as sensors, and cameras. Choosing the right equipment depends on the envisioned UAV application. Furthermore, a reliable data transmission system is required to share the collected data with the other UAVs and the ground infrastructure. The selection of the communication technology should be based on the region that an event occurs. This is because for example a UAV may be needed for an operation in a region that there is not any Base Station (BS) to support cellular systems. Therefore there should be a UAV that can communicate via Wi-Fi, WiMAX or SATCOM in consonance with the target region and the facilities onboard. Considering that each of the technologies has its own advantages and disadvantages. According to the communication technology, UAVs can construct different networking topologies such as mesh, and mobile/fly ad-hoc manner. Each of the UAVs is planned to accomplish a particular task. The tasks are assigned by the System Orchestrator (SO) which is the manager and the brain of the system architecture. The UAVs are piloted based on predetermined routes and plans so that they can fulfill their 
tasks. In addition, they are remotely controllable because they need to be turned on-and-off the sensors or cameras at the right time, in the right place. Figure 1 illustrates our envisioned UAV system architecture. It shows a heterogeneous network of different types of flying UAVs that are spread over a very large zone. UAVs can group various clusters in size to accomplish their designated tasks based on the application requirements and technology limitations.

The SO affords to benefit variety of Value-Added Services (VASs). These VASs can be built on the top of the deployed UAVs, rather than using the proprietary ones. Actually, every UAV that equipped with suitable and remotely controllable devices, like sensors and cameras, can offer these services. A dual-control UAV with a separate control on camera from the GS and UAVs with multi-functional UAVs (i.e. sensing the temperature or humidity while filming or taking photos) are good examples that provide VASs. A use case scenario is when a UAV is flying to deliver a package to a certain place where its path is predetermined. This UAV can pass above any places like roads, buildings and other predefined paths. Supposing that a transportation company (or an individual) needs to know the status of the traffic in a certain road and the UAV is currently flying above (or near) the intended road. Instead of using UAVs for the intended task(s), the company/individual can request from the owner of this UAV to collect the required data, e.g. taking a video from that road. Therefore, a triple benefit is achieved; for the traffic's company/individual as a costumer, for the owner of the UAV, and for the network operator.

Considering the VASs, lets assume that multiple UAVs are flying above a city and each UAV is planned for a specific task. Once in a certain region an event happens and the SO receives a request for a UAV to undertake an emergency task. In this case, the SO needs to decide which UAV is the best choice to assign the task to select it in a smart manner. This decision making and smart UAV selection, first of all, significantly depends on the equipment onboard, and then the energy level, the time required to complete the task, distance and the speed of the UAV. Studying these parameters is important in fulfilling of a task successfully and an efficient UAV task management.

\section{UAV SELECTION}

The initial consideration for selecting a UAV to do a specific task is subject to the equipment required by the task/event. Accordingly, the UAV residual energy amount and the energy requirement of the task is the effective parameter in the UAV selection process. Moreover, the distance to the event location, the UAV speed, the time needed to travel and complete a task; all need to be well investigated by developing optimized algorithms and computations in order to find an optimal solution for the best UAV selection. Another consideration is that a UAV selection is application-intended based, i.e. the type of application directly will affect the selection of the UAV. This is because the application specifies the type of required sensor(s), camera(s) or device(s) onboard. For example, once the $\mathrm{SO}$ receives a request that an event has happened in a

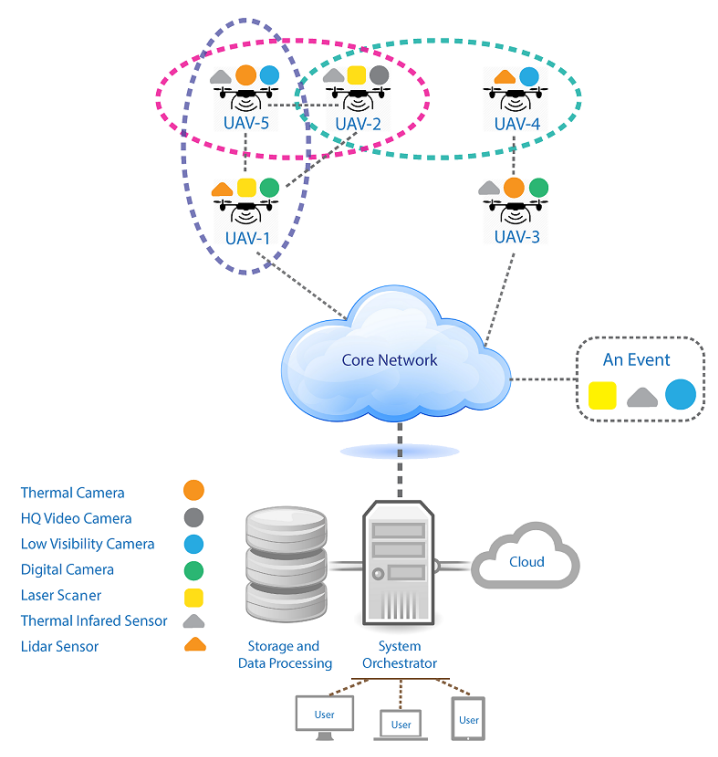

Fig. 1. $U A V$ network architecture and equipment-based $U A V$ set selection.

region on a certain location. Such as police needs an emergent photos from the scene of an accident. Moreover, the best UAV selection should be achieved, in case of minimizing the power consumption and required task operation time. For this reason, it is mandatory to seek an optimized way of selecting the best UAV for task accomplishment.

\section{A. Problem formulation for selection mechanism}

For UAV selection mechanism, let $u$ denote to a UAV, $\mathcal{N}$ be a set of UAVs in the network, $\mathcal{E}$ denote to an event, and $u \in \mathcal{N}$ i.e. $u$ be a UAV in $\mathcal{N}$. lets consider an example where five UAVs are flying and each one carries specific equipment onboard which pointed by different colors and shapes in figure 1. In the example of the accident and the photo required by the police from the scene. The SO should select a UAV that has the required facility i.e. Digital Camera. Therefore, there will be two options UAV1 and UAV3 that can undertake this task. For another example, once an event occurs and SO receives a request for a UAV which should have Laser Scanner, Low Visibility Camera and Thermal Infrared Sensor. Then, this makes the selection process more complex because UAVs do not obtain all the equipment onboard alone and it requires to apply an efficient selection mechanism. The information from the figure 1 shows that the possible solution to this should be a combination of these UAVs. This combination produces three sets of UAVs as $\left\{u_{1}, u_{5}\right\},\left\{u_{2}, u_{5}\right\}$, and $\left\{u_{2}, u_{4}\right\}$ where $u$ stands for UAV. After constructing the possible sets of UAVs, the best set should be chosen. The requirement of the best set selection is based on the computing the overall required time and energy to accomplish the task. The total time of the UAV operation depends on the travel time of UAV to the event location, sensing and processing and data transmission times. The total needed energy for task completion is computed based on the amount of the energy required for the travel, sensing/processing and the transmission energies. The optimal 
set of the UAVs will be the one that needs minimum amount of time and consumes the lowest amount of energy in order to accomplish and complete the task requested by the event.

\section{B. Energy consumption and required time for sensing and processing}

Any sensor which is applied with UAVs needs its own energy consumption amount and in some cases its own sampling frequency. Generally, it is assumed that a sensor has the following sensing energy consumption.

$$
\xi_{u}^{\text {Sensing }}=V_{d c} \times \mathrm{I}_{i} \times \mathrm{T}_{i}(1)
$$

Where, $T_{i}$ is the time needed for obtaining a single sample from the sensor $i$ and $I_{i}$ is the current draw of sensor. In order to compute the processing energy of the sensors $\xi_{u}^{\text {Processing, }}$ generally MCU's active and sleep mode for currents and times are used as in the following equation [5]:

$$
\begin{gathered}
\xi_{u}^{\text {Processing }}=V_{d c} \times \mathrm{I}_{m c u-a c t i v e} \times \mathrm{T}_{m c u-a c t i v e}+V_{d c} \times \mathrm{I}_{m c u-\text { sleep }} \\
\times \mathrm{T}_{\text {mcu-sleep }}(2)
\end{gathered}
$$

Then the energy and time of sense-and-process will be:

$$
\begin{gathered}
\xi_{u}^{\text {SensProcess }}=\xi_{u}^{\text {Sensing }}+\xi_{u}^{\text {Processing }} \text { (3) } \\
\Upsilon_{u}^{\text {SenseProcess }}=T_{i}+T_{m c u-\text { active }}(4)
\end{gathered}
$$

\section{The energy consumption and required time for traveling}

The major energy consumptions by the UAV is the amount of the energy that a UAV utilizes to travel to an event position. This energy is called the Traveling Energy of the UAV, $\xi_{u}^{\text {Travel }}$. In order to compute the travel energy, the travel distance $\mathcal{D}(u, \mathcal{E})$ between UAV and the event position should be calculated. With having the average velocity $\mathcal{V}_{u}$, the traveling time $\Upsilon_{u}^{\text {Travel }}$ can be computed. For calculating the travel energy $\xi_{u}^{\text {Travel}}$; coefficient $\lambda$, which represents the amount of energy consumption per one meter should be computed. $\lambda$ can be considered as the proportion of the full battery amount $\xi_{u}^{\text {Battery }}$ to $\mathcal{D}_{u}^{\text {Range }}$ which is the maximum distance that a UAV can fly. Considering $\mathcal{P}_{\mathcal{E}}=\left(X_{\mathcal{E}}, Y_{\mathcal{E}}, Z_{\mathcal{E}}\right)$ and $\mathcal{P}_{u}=\left(X_{u}, Y_{u}, Z_{u}\right)$; positions of an event and the position of a UAV in 3-D space respectively, and having $\Upsilon_{u}^{\text {Endurance, }}$ the UAV endurance time. The traveling time and energy of a UAV can be formulated as:

$$
\begin{gathered}
\mathcal{D}(u, \mathcal{E})=\sqrt{\left(X_{u}-X_{\mathcal{E}}\right)^{2}+\left(Y_{u}-Y_{\mathcal{E}}\right)^{2}+\left(Z_{u}-Z_{\mathcal{E}}\right)^{2}} \\
\Upsilon_{u}^{\text {Travel }}=\frac{\mathcal{D}(u, \mathcal{E})}{\mathcal{V}_{u}}(6) \\
\mathrm{D}_{u}^{\text {Range }}=\mathcal{V}_{u} \times \Upsilon_{u}^{\text {Endurance }}(7) \\
\lambda=\frac{\xi_{u}^{\text {Battery }}}{\mathcal{D}_{u}^{\text {Range }}}(8) \\
\xi_{u}^{\text {Travel }}=\lambda \times \mathcal{D}(u, \mathcal{E})(9)
\end{gathered}
$$

\section{Energy consumption and required time for Communication}

1) Communication Modeling: This section models the communications between a UAV $u$ and an eNodeB $\mathcal{B}$. In order to improve the communication reliability, an automatic repeat request (ARQ) scheme is used for forwarding the information. The ARQ scheme allows resending a packet until successful reception at the destination or a maximum number of retransmissions $M$ is reached. The number of ARQ retransmissions required for successful packet reception varies randomly according to the fading channel conditions.

Let $u$ denotes the transmitting $\mathrm{UAV}$, whereas $\mathcal{B}$ refer to the receiving eNodeB. The channel gain between $u$ and $\mathcal{B}$ is referred to as $\alpha_{u, \mathcal{B}}$. A Rayleigh block-fading channel is considered, where the channel gain $\alpha_{u, \mathcal{B}}$ remains constant over one block ${ }^{1}$ but changes independently from one block to another. The received signal $y_{\mathcal{B}}$ at a destination node $\mathcal{B}$ can be expressed as

$$
y_{\mathcal{B}}=\alpha_{u, \mathcal{B}} \sqrt{P_{u}} x_{u}+n_{\mathcal{B}},
$$

where $P_{u}$ denotes transmission powers of UAV $u$. The symbols transmitted by node $u$ is referred to as $x_{u} . n_{\mathcal{B}}$ is a zero-mean additive white Gaussian noise with variance $N_{0}$. The term $\gamma_{u, \mathcal{B}}$ denotes the instantaneous received signal-to-noise ratio at $\mathcal{B}$ given by $\gamma_{u, \mathcal{B}}=P_{u} \alpha_{u, \mathcal{B}}^{2} / N_{0}$ [6]. The mean value of $\gamma_{u, \mathcal{B}}$ is denoted as $\bar{\gamma}_{u, \mathcal{B}}$ which can be expressed as

$$
\bar{\gamma}_{u, \mathcal{B}}=\frac{P_{u} \mathbb{E}\left[\alpha_{u, \mathcal{B}}^{2}\right]}{N_{0}}
$$

where $\mathbb{E}\left[\alpha_{u, \mathcal{B}}^{2}\right]$ represents the channel variance, and $\mathbb{E}[\cdot]$ is the expectation operator. Using a distance dependent path loss model, the channel variance can be determined as [7]

$$
\mathbb{E}\left[\alpha_{x, y}^{2}\right]=\left(\frac{\mathcal{D}_{0}}{\mathcal{D}_{u, \mathcal{B}}}\right)^{\beta}
$$

where $\mathcal{D}_{u, \mathcal{B}}$ refers to the distance between nodes $u$ and $\mathcal{B}$, $\mathcal{D}_{0}$ denotes a reference distance typically set to $1 \mathrm{~m}$, and $\beta$ denotes the path loss exponent. In our physical model, we take into account the effects of both path loss and fast fading, while the impact of shadowing is neglected.

Theorem 1. For any $U A V u \in \mathcal{N}$ fails to transmit its packet to an eNodeB $\mathcal{B}$ iff $\mathrm{SNR}_{u, \mathcal{B}}$ falls below a threshold $\gamma_{\mathrm{th}}$. This event is known as an outage event and occurs with a probability $\mathcal{P}_{u, \mathcal{B}}$ which can be expressed as

$$
\mathcal{P}_{u, \mathcal{B}}=1-\exp \left(-\frac{\gamma_{\text {th }}}{\bar{\gamma}_{u, \mathcal{B}}}\right) .
$$

Proof. In this appendix, we derive the proof for the outage probability between $u$ and $\mathcal{B}$ in the network. By definition, the link $u-\mathcal{B}$ is in outage if $\mathrm{SNR}_{u, \mathcal{B}}$ falls below a threshold level $\gamma_{t h}$ [6]. This event occurs with a probability $\mathcal{P}_{u, \mathcal{B}}$. In order to determine an expression for the outage probability $\mathcal{P}_{u, \mathcal{B}}$, we need first to compute the CDF of $\mathrm{SNR}_{u, \mathcal{B}}$.

First, we recall that the expression of the SNR is given by

$$
\mathrm{SNR}_{u, \mathcal{B}}=\gamma_{u, \mathcal{B}}
$$

\footnotetext{
${ }^{1} \mathrm{~A}$ block corresponds to the time duration necessary to send one packet.
} 
where $\gamma_{u, \mathcal{B}}$ is exponential random variables with mean values $\bar{\gamma}_{u, \mathcal{B}}$. The probability density functions (PDFs) of $\gamma_{u, \mathcal{B}}$ is:

$$
p_{\gamma_{u, \mathcal{B}}}(z)=\frac{1}{\bar{\gamma}_{u, \mathcal{B}}} \exp \left(-\frac{z}{\bar{\gamma}_{u, \mathcal{B}}}\right),
$$

The random variable $\gamma_{u, \mathcal{B}}$ follows an exponential distribution and its $\mathrm{CDF}$ is given by

$$
\begin{gathered}
F_{\gamma_{u, \mathcal{B}}}(z)=1-\exp \left(-\frac{z}{\bar{\gamma}_{u, \mathcal{B}}}\right), \\
\mathcal{P}_{u, \mathcal{B}}=P\left(\mathrm{SNR}_{u, \mathcal{B}} \leq \gamma_{\mathrm{th}}\right)=P\left(\gamma_{u, \mathcal{B}} \leq \gamma_{\mathrm{th}}\right) \\
=F_{\gamma_{u, \mathcal{B}}}(z)=1-\exp \left(-\frac{\gamma_{\mathrm{th}}}{\bar{\gamma}_{u, \mathcal{B}}}\right) .
\end{gathered}
$$

2) Communication time modeling: The UAVs be equipped with a buffer to store the packets before their transmission. We assume that UAV has $\mathcal{K}$ packets that represent the sensed information about an event $\mathcal{E}$. This section focuses on the analysis of the average delay of transmission $\Upsilon_{u}^{\text {Transmit }}$ of sensed data from $u$ to an eNodeB $\mathcal{B}$. We do not take into account neither the time required for sensing and processing nor the time required for traveling from the event $\mathcal{E}$ to $\mathcal{B}$. Let $T_{u}^{\text {Transmit }}$ represents the sojourn time of a packet before its transmission to $\mathcal{B}$. Formally, $\Upsilon_{u}^{\text {Transmit }}=\mathcal{K} \cdot T_{u}^{\text {Transmit }}$. A successful reception of a packet at eNodeB $\mathcal{B}$ occurs after a random number of retransmissions. To quantify the delay associated with the retransmission events, we measure the average sojourn time $T_{u}^{\text {Transmit }}$ of a packet in the buffer of $u$, which is defined as the average time elapsed from the starting of its transmission until its successful reception. The packet's sojourn time in the buffer can be evaluated using the PollaczekKhinchin equation as [8],

$$
T_{u}^{\text {Transmit }}=\mathbb{E}\left(N_{u, \mathcal{B}}\right) T_{F},
$$

where $T_{F}$ is the time required for a single transmission of a given packet and $\mathbb{E}\left(N_{u, \mathcal{B}}\right)$ is the average number of retransmissions for the packets sent by $u$. For the ARQ scheme, the packet is retransmitted until successful reception at the $\mathcal{B}$ or a maximum number of retransmissions $M$ is reached. In case of reception failure after $M$ retransmissions the packet is discarded. The number of retransmissions $N_{u, \mathcal{B}}$ varies randomly according to the position of UAV and the conditions of the fading channel between the $u$ and $\mathcal{B}$. The average number of retransmissions $\mathbb{E}\left(N_{u, \mathcal{B}}\right)$ can be expressed as [9],

$$
\begin{aligned}
\mathbb{E}\left(N_{u, \mathcal{B}}\right) & =1+\sum_{m=1}^{M-1} P\left(F^{1}, \ldots, F^{m}\right)=1+\sum_{m=1}^{M-1}\left(\mathcal{P}_{u, \mathcal{B}}\right)^{m} \\
& =\sum_{m=0}^{M-1}\left(\mathcal{P}_{u, \mathcal{B}}\right)^{m}=\frac{1-\left(\mathcal{P}_{u, \mathcal{B}}\right)^{M}}{1-\mathcal{P}_{u, \mathcal{B}}}
\end{aligned}
$$

where $P\left(F^{1}, \ldots, F^{m}\right)$ is the probability of a reception failure at the $1, \ldots, m$ th retransmissions. Since the channel realizations in each transmission are independent identically distributed, the event of reception failure at each step are independent and have equal probabilities, thus $P\left(F^{1}, \ldots, F^{m}\right)=\left(\mathcal{P}_{u, \mathcal{B}}\right)^{m}$.

From 18 and 19, we have:

$$
\Upsilon_{u}^{\text {Transmit }}=\mathcal{K} \cdot T_{F} \cdot \mathbb{E}\left(N_{u, \mathcal{B}}\right) \frac{1-\left(\mathcal{P}_{u, \mathcal{B}}\right)^{M}}{1-\mathcal{P}_{u, \mathcal{B}}} .
$$

3) Energy consumption modeling in communication: This section studies the energy consumption at UAV $u$. Lets assume that UAV has $\mathcal{K}$ packets that represent the sensed information about an event $\mathcal{E}$. The fact that the number of retransmissions varies depending on the channel conditions makes the consumed power a random variable. This section studies the average consumed power and then deduce from that the average consumed energy. The average consumed power $\bar{P}$ for the ARQ scheme can be determined as

$$
\begin{aligned}
\bar{P} & =P_{u} \cdot P\left(S^{1}\right)+2 P_{u} \cdot P\left(F^{1}, S^{2}\right)+\ldots \\
& +(M-1) P_{u} \cdot P\left(F^{1}, \ldots, S^{M-1}\right)+M P_{x} \cdot P\left(F^{1}, \ldots, F^{M-1}\right) \\
& =P_{u} \cdot\left(1+\sum_{m=1}^{M-1} P\left(F^{1}, \ldots, F^{m}\right)\right)=P_{u} \cdot\left(1+\sum_{m=1}^{M-1}\left(\mathcal{P}_{u, \mathcal{B}}\right)^{m}\right) \\
& =P_{u} \cdot \mathbb{E}\left(T_{u, \mathcal{B}}\right)=P_{u} \cdot \frac{1-\left(\mathcal{P}_{u, \mathcal{B}}\right)^{M}}{1-\mathcal{P}_{u, \mathcal{B}}}
\end{aligned}
$$

where the term $P_{u}$ stands for the power per retransmission at a given $u$. We denote by $P\left(S^{1}\right)$ the probability of successful reception at $\mathcal{B}$ of the first transmission, while $P\left(F^{1}, \ldots, S^{M-1}\right)$ refers to the probability of a reception failure in the 1 st, 2 nd, $\ldots,(M-2)$ th retransmissions and a successful reception at the $(M-1)$ th retransmission. If the packet is successfully received after the first transmission (this event occurs with a probability $P\left(S^{1}\right)$ ), the amount of consumed power would be equal to $P_{u}$. If the packet is received correctly after two retransmissions (the probability of this event is $P\left(F^{1}, S^{2}\right)$ ), the amount of consumed power would be equal to $2 P_{u}$. The consumed power would be equal to $M P_{u}$ if the 1 st, $\ldots,(M-1)$ th retransmissions fails (the probability of this event is $\left.P\left(F^{1}, \ldots, F^{M-1}\right)\right)$. The average consumed power is obtained by summing up all the possible values of consumed power weighted by their respective probability of occurrence. The result in (21) shows that we can express the average consumed power as the product of two terms: the power per retransmission $P_{u}$ and the average number of retransmissions $\mathbb{E}\left(T_{u, \mathcal{B}}\right)$. The average consumed energy $\Phi_{u, \mathcal{B}}$ of one packet can be obtained as

$$
\begin{aligned}
\Phi_{u, \mathcal{B}} & =P_{u} \cdot T_{F} \cdot P\left(S^{1}\right)+2 P_{u} \cdot T_{F} \cdot P\left(F^{1}, S^{2}\right)+\ldots+(M-1) P_{u} \\
& \cdot T_{F} \cdot P\left(F^{1}, \ldots, S^{M-1}\right)+M P_{u} \cdot T_{F} \cdot P\left(F^{1}, \ldots, F^{M-1}\right) \\
& =P_{u} \cdot T_{F} \cdot\left(1+\sum_{m=1}^{M-1}\left(\mathcal{P}_{u, \mathcal{B}}\right)^{m}\right)=P_{u} \cdot T_{F} \cdot \mathbb{E}\left(T_{u, \mathcal{B}}\right)=\bar{P} \cdot T_{F} .
\end{aligned}
$$

The average consumed energy $\xi_{u}^{\text {Transmit }}$ to transmit the whole data ( $\mathcal{K}$ packets) can be obtained as

$$
\xi_{u}^{\text {Transmit }}=\mathcal{K} \cdot \Phi_{u, \mathcal{B}} \quad=\mathcal{K} \cdot \bar{P} \cdot T_{F} .
$$

\section{OPTIMAL SOLUTIONS FOR UAV SELECTION}

Let we denote by $\xi_{u}^{T o T}$ and $\Upsilon_{u}^{T o T}$, the total energy and the total time required by a UAV $u$ to accomplish a mission for handling an event $\mathcal{E}$. Formally,

$$
\begin{gathered}
\xi_{u}^{T o T}=\xi_{u}^{\text {Travel }}+\xi_{u}^{\text {SenseProcess }}+\xi_{u}^{\text {Transmit }}, \\
\Upsilon_{u}^{T o T}=\Upsilon_{u}^{T \text { ravel }}+\Upsilon_{u}^{\text {SenseProcess }}+\Upsilon_{u}^{\text {Transmit }},
\end{gathered}
$$


Here, we propose two Linear Integer Problem to deal the problem of UAV selection. In the first optimization problem, we aim to minimize as much as possible the energy consumption to handle the event $\mathcal{E}$. This solution aims to ensure a fair energy consumption among different UAVs. Meanwhile, the second optimization problem aims at minimizing the time to handle an event regardless of the energy consumption. Before starting the selection process, the eligible UAVs should be selected. Let $\ddot{\mathcal{N}}$ denotes the eligible UAVs in the network. Formally, $\ddot{\mathcal{N}}$ is defined as the UAVs that satisfy the following conditions:

- Power constraint: The selected UAVs should have the enough power to undertake a task, i.e. $\xi_{u}^{\text {Battery }}>\xi_{u}^{T o T}$;

- Equipment constraint: This constraint ensures that the eligible UAV can attend the event $\mathcal{E}$ and it has the required sensors for the operation. Formally this constraint is defined by the following conditions:

- A UAV has the ability of flying that allows it to reach the altitude of the event $\mathcal{E}$. Formally, $m \mathcal{H}_{u} \geq Z_{\mathcal{E}}$; $m \mathcal{H}_{u}$ is the maximum altitude that a UAV can fly.

- A UAV has the required sensors to handle the event $\mathcal{E}$. Formally, $\mathcal{S}_{u} \cap \mathcal{S}_{\mathcal{E}} \neq \emptyset$. $\mathcal{S}_{\mathcal{E}}$ is the sensor/s required by the event and $\mathcal{S}_{u}$ refers to UAV Sensor/s onboard.

\section{A. Optimization of energy consumption}

Let $\mathcal{X}_{u}$ be a boolean decision variable that represents if a UAV $u \in \ddot{\mathcal{N}}$ should be selected to handle the event $\mathcal{E}$.

$$
\mathcal{X}_{u}= \begin{cases}1 & \text { If } u \text { is selected to handle the event } \mathcal{E} \\ 0 & \text { Otherwise }\end{cases}
$$

This solution aims to minimize as much as possible the energy consumption by selecting the minimum number of UAVs in the network while the time delay do not exceed a predefined threshold $\Upsilon_{t h}$. This solution is formulated through the following Linear Integer Problem:

$$
\left\{\begin{array}{l}
\min \sum_{u \in \ddot{\mathcal{N}}} \xi_{u}^{T o T} \cdot \mathcal{X}_{u} \\
\text { s. t. } \\
\forall s \in \mathcal{S}_{\mathcal{E}}: \sum_{u \in \ddot{\mathcal{N}} \wedge s \in \mathcal{S}_{u}} \mathcal{X}_{u} \geq 1 \\
\forall u \in \ddot{\mathcal{N}}: \Upsilon_{u}^{T o T} \cdot \mathcal{X}_{u} \geq \Upsilon_{t h} \\
\forall u \in \ddot{\mathcal{N}}: \mathcal{X}_{u} \in\{0,1\}
\end{array}\right.
$$

Where, the first constraint ensures that the selected UAVs have the required sensors to deal with the event $\mathcal{E}$. The second constraint ensures that the time latency of each selected UAV should not exceed the threshold $\Upsilon_{t h}$. The last constraint ensures that $\mathcal{X}_{u}$ is a boolean decision variable.

\section{B. Optimization of operation time}

Let $\mathcal{X}_{u}$ be a boolean decision variable that represents if a UAV $u \in \ddot{\mathcal{N}}$ should be selected to handle the event $\mathcal{E}$.

$$
\mathcal{X}_{u}= \begin{cases}1 & \text { If } u \text { is selected to handle the event } \mathcal{E} \\ 0 & \text { Otherwise }\end{cases}
$$

This solution aims to minimize as much as possible the time response of UAVs while the residual energy of these UAVs should exceed a predefined threshold $\xi_{t h}$. This solution is formulated through the following Linear Integer Problem:

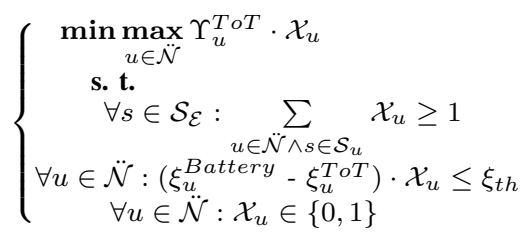

Where, the first constraint ensures that the selected UAVs have the required sensors to deal with the event $\mathcal{E}$. The second constraint ensures that the residual energy in each selected UAV should be higher than the $\xi_{t h}$. The last constraint ensures that $\mathcal{X}_{u}$ is a boolean decision variable.

\section{Simulation results}

For our proposed optimal solutions, we developed a simulator using Python and implemented through Gurobi optimization tool. In the simulations, we call the solutions as Energy Aware Selection of UAVs (EAS) and Delay Aware Selection of UAVs (DAS). In the simulation results, each plotted point represents the average of 200 times of executions. The plots are presented with $95 \%$ confidence interval. The applied algorithm evaluates the performance of the UAV selection in terms of the number of UAVs and the size of UAVs flying area. For both cases the total energy consumption and the operation time is considered. It is assumed that there is only one event has happened which requires 6 different type of sensors and UAVs have variant number of sensors onboard. In the simulations, performances of EAS and DAS are evaluated two times. First by varying number of UAVs with fixed area size equal to 10000 square meters. Second evaluation made by varying area size with fixed number of UAVs equal to 150 .

1) Performances evaluation by varying number of UAVs: For this evaluation, with the objective of total energy consumption, Fig.2(a) represents the number of UAVs in the network and the total energy consumption by the attending UAVs. It shows increasing number of UAVs in the network and with this increment, the availability of the required number of sensors for the event increases. This means that a selection, i.e. set of UAVs jointly can accomplish the task. By increasing the number of the UAVs the required amount of energy for the operation is decreasing. For example a set with 250 UAVs, requires $100 \mathrm{mAh}$ per $\mathrm{UAV}$ to do the task in the EAS manner while it is more efficient and consumes less power (nearly 200 mAh per UAV) compared to the DAS. With the objective of operation time, Fig.2(b) explains that by increasing the number of the UAVs in the network, the required operation time for participating in an even is considerably decreasing. In addition, in the comparison DAS shows much better performance than the EAS optimization in terms of operation time.

2) Performances evaluation by increasing the size of flying Area: For this evaluation, with the objective of total energy consumption, Fig.3(a) demonstrates the size of UAVs flying area with the high performance of EAS toward DAS in terms of total energy consumption. This is because, for example if 


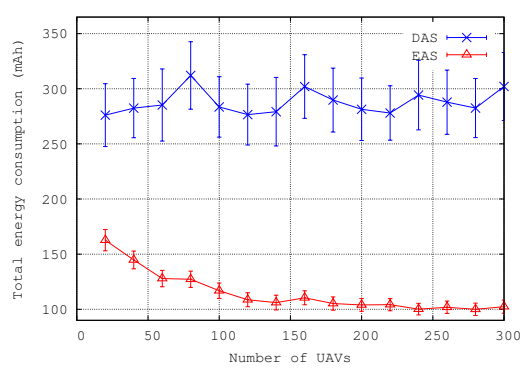

(a) The energy consumption

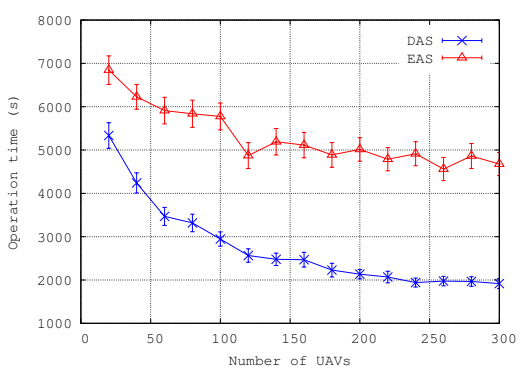

(b) The operation time

Fig. 2. Performances evaluation by varying the number of UAVs

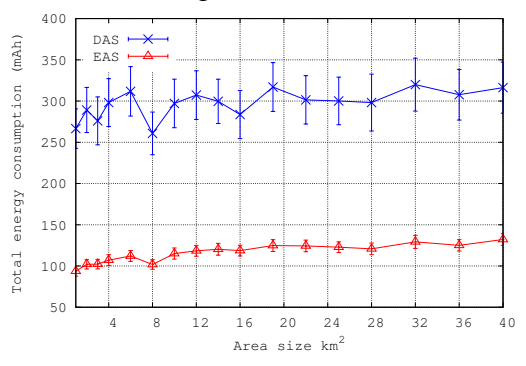

(a) The energy consumption

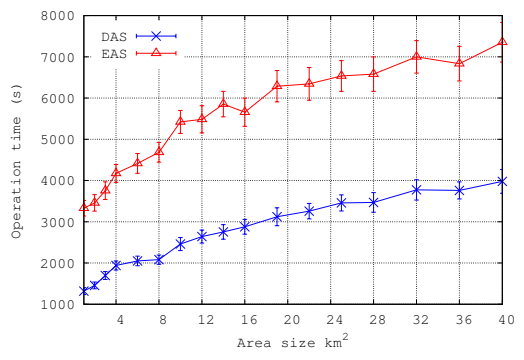

(b) The operation time

Fig. 3. Performances evaluation by increasing the size of flying Area

all the UAVs are flying in an area of $25 \mathrm{Km}^{2}$, in order to do the task they consume $125 \mathrm{mAh}$ per UAV in EAS manner while they must consume $300 \mathrm{mAh}$ per UAV in DAS manner. This illustrates that EAS has more efficiency than DAS where the size of operation area is increasing. With the objective of operation time, Fig.3(b) explains that by increasing the flying area of UAVs the required operation time for participating in an event is slightly increasing and in the comparison, DAS shows better performance than the EAS optimization.

\section{CONCLUSION}

In near future billions of IoT devices such as sensors, and cameras that are installed on the earth or are on the fly like UAVs onboard will be connected. The UAVs can construct a communication network in an ad-hoc manner in a way that some of them are on the fly and others are ready to fly when needed. Each of these UAVs can be assigned to accomplish a particular task. Employing UAVs can offer a variety of Value-Added IoT Services. These VASs can be built on the top of the deployed UAVs, rather than using the proprietary ones. Selecting the best UAV from a set of flying ones, is subject to the capability of the UAV in terms of obtaining the required equipment onboard and the current residual energy of battery of the UAV. In this paper, we referred to our envisioned UAV communication platform and we discussed the different components needed to build a realistic UAV-based IoT system. For an efficient UAV selection mechanism we provided the formulation that results in the best UAV set selection from among the all sets. For the solutions we proposed two optimization problems using Linear Integer Problem to select the optimal set of UAVs. For the solutions and the result of our optimization work, we presented Energy Aware Selection of
UAVs (EAS) and Delay Aware Selection of UAVs (DAS) that evaluated through the simulations. For these solutions UAV selection is simulated in terms of the number of the UAVs and the size of the UAVs flying area. For both cases the total energy consumption and the operation time is considered. The result of this simulation depicts that; in UAV selection if the operation time is the target to achieve, DAS which aims to minimize as much as possible the response times of UAVs should be applied. Additionally, in UAV selection if the objective is the energy consumption by UAVs; EAS that aims to minimize as much as possible the energy consumption should be used.

\section{REFERENCES}

[1] V. Ovidiu and F. Peter, Internet of Things: Converging Technologies for Smart Environments and Integrated Ecosystems. River Publishers, 2013.

[2] C. Guowei, D. Jroge, and S. Lakma, "A Survey of Small-Scale Unmanned Aerial Vehicles: Recent Advances and Future Development Trends," Unmanned Systems, vol. 2, no. 2, p. 125, Feb. 2014.

[3] A. Ricardo. (2016) Delivery drones via air. [Online]. Available: http://unmannedcargo.org/

[4] F. Phil. (2015) Aerospace and Defence Market Intelligence Analysis and Forcasts, Missile and UAV Market Forecasts. [Online]. Available: http://www.tealgroup.com/

[5] A. R. Mohammad and D. Simon, "Energy-Efficient Sensing in Wireless Sensor Networks Using Compressed Sensing," Sensors, vol. 14, no. 2, pp. 2822-2859, Feb. 2014.

[6] M. K. Simon and M.-S. Alouini, Digital Communication over Fading Channels, 2nd ed. New Jersey, USA: John Wiley \& Sons, 2005.

[7] A. F. Molisch, Wireless Communications. Wiley-IEEE Press, 2005.

[8] W. Chan, T.-C. Lu, and R.-J. Chen, "Pollaczek-Khinchin formula for the M/G/1 queue in discrete time with vacations," IEE Proceedings Computers and Digital Techniques, vol. 144, no. 4, pp. 222-226, Jul. 1997.

[9] A. Chelli, E. Zedini, M.-S. Alouini, J. R. Barry, and M. Pätzold, "Performance and delay analysis of hybrid ARQ with incremental redundancy over double Rayleigh fading channels," IEEE Trans. on Wireless Commun., vol. 13, no. 11, pp. 6245-6258, Nov. 2014. 